\title{
Causal Relationships between CSI300 Spot and Its Index Futures Revealed by Granger Causality and New Causality
}

\author{
Quanquan Liu ${ }^{1, a}$, Sanqing $\mathrm{Hu}^{1, \mathrm{~b}}$, Jianhai Zhang ${ }^{1, \mathrm{c}}$, Wanzeng Kong ${ }^{1, \mathrm{~d}}$ \\ ${ }^{1}$ Compute college, Hang zhou Dianzi University, Hang Zhou, China

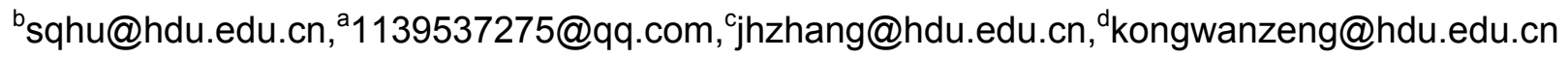

Keywords: Granger Causality, New Causality, CSI 300 Spot, CSI 300 Index Futures

\begin{abstract}
Granger causality has been widely applied to stock markets to study causal relationships between two time series variables. In this paper, we use Granger Causality (GC) and our recently proposed New Causality (NC) to find the causality relationship between the CSI 300 Spot and its index futures which covers the time period from April 7, 2015 to September 8, 2015. The stock market in this period in China experienced Crazy rise and rapid crash. We first use GC and NC methods to analyze the causality between the CSI 300 Spot and its index future for the all sample period. The results by both methods reveal that the causal influence from CSI 300 index futures to CSI 300 Spot is much greater than that from CSI 300 Spot to CSI 300 index futures. We then calculate the rolling causality using two methods. The GC results show that the causal influence from CSI 300 index futures to CSI 300 Spot is always much greater than that from CSI 300 Spot to CSI 300 index, this may not be true in practice. The NC results show that the causal influence from CSI 300 index futures to CSI 300 Spot is greater than that from CSI 300 Spot to CSI 300 index by $80 \%$, this may be true in practice. Anyway, both methods demonstrate that CSI 300 index futures has a major causal influence in CSI 300 Spot.
\end{abstract}

\section{Introduction}

Causality is used for identifying the direction of information flow for over two thousand years. Researchers proposed many causality methods to reveal causal influence in one system. In the literature, one of the most popular definition is Granger Causality (GC) [1]. It has been widely used in many different areas, such as economic and climate studies and genetics and neuroscience. The basic idea of causality can be traced back to Wiener[2] who conceived the notion that, if the prediction of one time series could be improved by incorporating the knowledge of a second one, then the second series is said to have a causal influence on the first. Granger later formalized the prediction idea in the context of linear regression models. Specifically, if the variance of the autoregressive prediction error of the first time series at the present time is reduced by inclusion of past measurements from the second time series, then the second time series is said to have a causal influence on the first one. The roles of the two time series can be reversed to address the question of causal influence in the opposite direction [3], Although GC is applied to many different areas, it has also been criticized by many researchers from many aspects. In $2011, \mathrm{Hu}$ et.al pointed out many shortcomings/limitations of GC by using many illustrative examples and showed that GC is only a causality definition in the sense of Granger and why GC does not reflect real causality and proposed new causality (NC). The basic idea of $\mathrm{NC}$ is to use the proportion that $\mathrm{Y}$ occupies among all contributions to $\mathrm{X}$ to describe the strength of the real causality form $\mathrm{Y}$ to $\mathrm{X}$ which is much better than GC in many aspects [4].

There is a large body of research dedicated to investigating the causality between prices in the futures market and the spot market[5][6][7]. However, most of these studies use GC definition to calculate causality and attempt to find a lead-lag relationship between the stock index price and the underlying prices of futures contracts.

In this paper, we firstly give GC and NC methods in time domain and then we use GC and NC to analyze the causality between CSI 300 Spot and its index futures over the entire sample period in time domain. Secondly we calculate the rolling causality[8] using GC and NC methods. Both methods demonstrate that CSI 300 index futures has a major causal influence in CSI 300 Spot. 


\section{Causality Methods}

\subsection{GC and NC Methods}

Consider two stochastic time series which are assumed to be jointly stationary. Individually, under fairly general conditions, each time series admits an autoregressive representation

$$
\left\{\begin{array}{l}
\mathrm{X}_{1, \mathrm{t}}=\sum_{j=1}^{m} a_{11, j} X_{1, t-j}+\varepsilon_{1, t} \\
X_{2, t}=\sum_{j=1}^{m} a_{22, j} X_{1, t-j}+\varepsilon_{2, t}
\end{array}\right.
$$

and their joint representations are described as:

$$
\left\{\begin{array}{l}
\mathrm{X}_{1, t}=\sum_{j=1}^{m} a_{11, j} X_{1, t-j}+\sum_{j=1}^{m} a_{12, j} X_{2, t-j}+\eta_{1, t} \\
X_{2, t}=\sum_{j=1}^{m} a_{21, j} X_{1, t-j}+\sum_{j=1}^{m} a_{22, j} X_{2, t-j}+\eta_{2, t}
\end{array}\right.
$$

where $\mathrm{t}=0,1, \ldots, \mathrm{N}$, the noise terms are uncorrelated over time, $\varepsilon_{i}$ and $\eta_{i}$ have zero means and variances of $\sigma_{\varepsilon_{i}}^{2}$ and $\sigma_{\eta_{i}}^{2}, \mathrm{i}=1,2$, The covariance between $\eta_{1}$ and $\eta_{2}$ is defined by $\sigma_{\eta_{1} \eta_{2}}=\operatorname{cov}\left(\eta_{1}, \eta_{2}\right)$.

\subsubsection{Granger Causality In Bivariate Autoregressive Model}

Now consider the first equalities in (1) and (2), if $\sigma_{\eta_{i}}^{2}$ is less than $\sigma_{\varepsilon_{i}}^{2}$ in some suitable sense $\mathrm{X}_{2}$ is said to have a causal influence on $X_{1}$. In this case, the first equality in (2) is more accurate than in (1) to estimate $x_{1}$ Otherwise, if $\sigma_{\eta_{i}}^{2}=\sigma_{\varepsilon_{i}}^{2}, x_{2}$ is said to have no causal influence on $X_{1}$. In this case, two equalities are same. Such kind of causal influence, called GC, is defined by

$$
\mathrm{F}_{X_{2} \rightarrow X_{1}}=\ln \frac{\sigma_{\varepsilon_{1}}^{2}}{\sigma_{\eta_{1}}^{2}}
$$

Obviously, $\mathrm{F}_{X_{2} \rightarrow X_{1}}=0$ when there is no causal influence from $X_{2}$ to $X_{1}$ and $\mathrm{F}_{X_{2} \rightarrow X_{1}}>0$ when there is. Similarly, the causal influence from $\mathrm{x}_{2}$ to $x_{1}$ is defined by

$$
\mathrm{F}_{X_{1} \rightarrow X_{2}}=\ln \frac{\sigma_{\varepsilon_{2}}^{2}}{\sigma_{\eta_{2}}^{2}}
$$

\subsubsection{New Causality In Bivariate Autoregressive Model}

Based on (2), we can see contributions to $x_{1}$, which include $\sum_{j=1}^{\mathrm{m}} a_{1, j} X_{1, t-j}, \sum_{j=1}^{\mathrm{m}} a_{2, j} X_{2, t-j}$ and the noise term $\eta_{1, t}$ where the influence from $\sum_{j=1}^{\mathrm{m}} a_{1, j} X_{1, t-j}$ is causality from $\mathrm{X}_{1}$ own past values. Each contribution plays an important role in determining $X_{1}$, If $\sum_{j=1}^{m} a_{21, j} X_{2, t-j}$ occupies a larger portion among all those contributions, then $x_{2}$ has stronger causality on $x_{1}$ or vice versa. Thus, a good definition for causality from $X_{2}$ to $X_{1}$ in time domain should be able to describe what proportion $X_{2}$ occupies among all these contributions.

So based on this general guideline New causality from $X_{2}$ to $X_{1}$ is defined as

$$
n_{X_{2} \rightarrow X_{1}}=\frac{\sum_{t=m}^{N}\left(\sum_{j=1}^{m} a_{12, j} X_{2, t-j}\right)^{2}}{\sum_{h=1}^{n} \sum_{t=m}^{N}\left(\sum_{j=1}^{m} a_{1 h, j} X_{h, t-j}\right)^{2}+\sum_{t=m}^{N} \eta^{2}{ }_{1, t}}
$$

Similarly, new causality in time domain from $X_{1}$ to $x_{2}$ is defined by

$$
n_{X_{1} \rightarrow X_{2}}=\frac{\sum_{t=m}^{N}\left(\sum_{j=1}^{m} a_{21, j} X_{1, t-j}\right)^{2}}{\sum_{h=1}^{n} \sum_{t=m}^{N}\left(\sum_{j=1}^{m} a_{2 h, j} X_{h, t-j}\right)^{2}+\sum_{t=m}^{N} \eta^{2}{ }_{2, t}}
$$




\section{Experimental Method}

We select the time period from April 7, 2015 to September 8, 2015 as the sample period. Two time series are employed in our analysis. One consists of the closing minute prices of the CSI 300 Spot and the other consists of the closing minute prices of the closely related CSI 300 index futures contract, which are characterized by high liquidity and activity. The data are obtained from the Wind Info [9]. The spot data is defined as $\operatorname{smean}_{\mathrm{t}}=S_{t}-$ mean $(S)$, where $\mathrm{S}_{t}$ is the price in the spot market at time (minute) $t$, while the futures data is defined as fmean $=F_{t}-$ mean $(F)$, where $\mathrm{F}_{t}$ is the futures price of the nearby contract at time (minute) $t$.

\section{The Simulation Results}

In this section, we use GC and NC methods to calculate the causality between CSI300 Spot and its Index Futures. Firstly we use all the dataset to calculate causality and secondly we calculate the rolling causality by using moving window technology where the window size is set to be 1500 .

\subsection{Causality performed over the entire sample period}

We first calculate causality values between CSI300 Spot and its future index for the whole sample period and get GC value from CSI300 Index Future to CSI300 Spot is 0.1378 and GC value from CSI300 Spot to CSI300 Index Future is 0.0054, We also obtain NC value from CSI300 Index Future to CSI300 Spot is 2.4691e-05 and NC value from CSI300 Spot to CSI300 Index Future is 1.2787e-0.6. Therefore, Both methods reveal that CSI300 Index Future have a major causal influence on CSI300 Spot. To show our causality values are of significance, next we shuffle CSI 300 Index Futures by 100 times, calculate the causality value from CSI 300 Index Futures (surrogate data) to CSI300 Spot, and obtain 100 causality values for GC method or NC method. The results are shown in Figure 1 and Figure 2 respectively where blue points are causality value from CSI 300 Index Futures (raw data) to CSI300 Spot (raw data). From Figure 1 and Figure 2 one can see that GC or NC value from CSI 300 Index Futures (raw data) to CSI300 Spot (raw data) is significantly different from that from CSI 300 Index Futures (surrogate data) to CSI300 Spot (raw data). Therefore, GC value or NC value is of significance.
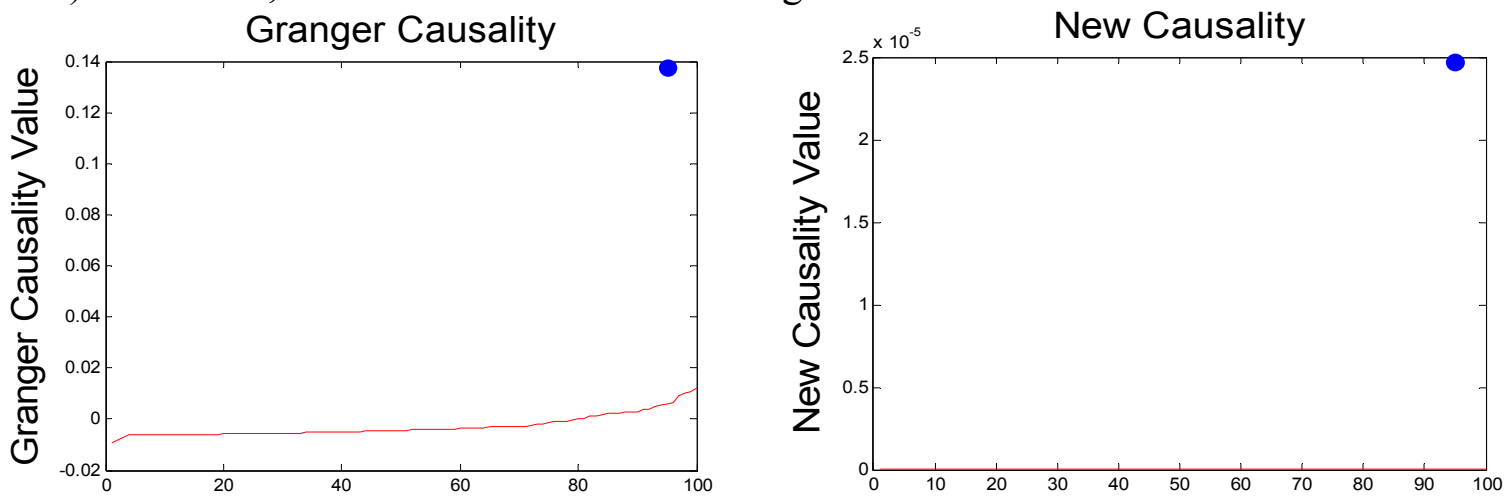

Figure1.Granger Causality for all sample period Figure 2. New Causality for all sample period

\subsection{Rolling causality performed over moving windows}

In this section, we calculate the rolling causality by moving window technology where the length of each window is set to be 1500 and two neighbor windows overlap is 1490 . For each window we calculate GC and NC values. The results are reported in Figure 3 and Figure 4.

In Figure 3 and Figure 4, the blue curve represents GC and NC values from CSI300 Spot to CSI300 Index Future respectively. The red curve represents GC and NC values from CSI 300 Index Future to CSI300 Spot respectively. From Figure 3 one can see that the red curve is always on the top of the blue curve by using GC method. This means CSI300 Index Future always has major 
causal influence on CSI300 Spot. In practice this phenomenon is not true because stock market is of high randomness. From Figure 4 we can see that the causal influence from CSI 300 index futures to CSI 300 Spot is greater than that from CSI 300 Spot to CSI 300 index by $80 \%$. This is more consistent with practice.

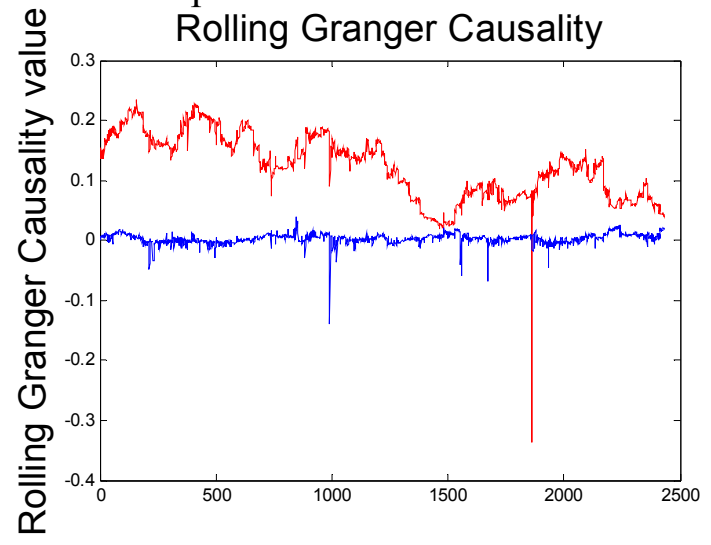

Figure3.Rolling Granger Causality

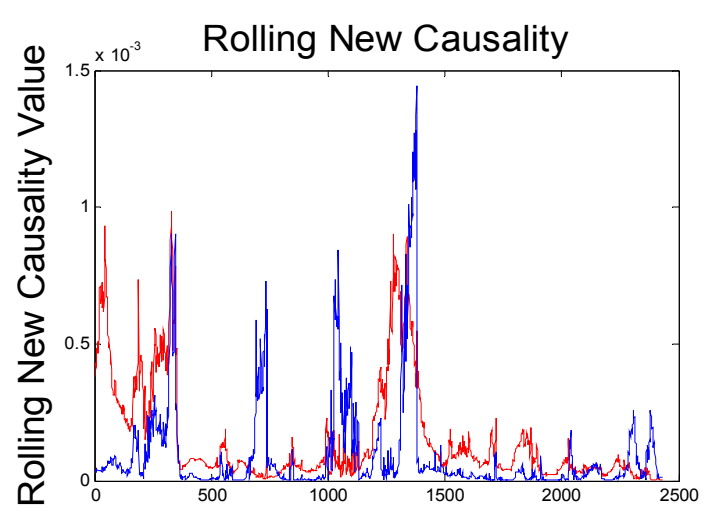

Figure4.Rolling New Causality

\section{Conclusion}

In this paper, we used GC and NC methods to calculate the causality between CSI300 Spot and its Index Futures. Firstly we used the whole sample points to calculate causality, and drew the conclusion that both methods reveals that CSI300 Index Future has a major causal influence on CSI300 Spot. We then calculated the rolling causality using two methods. The GC results revealed that the causal influence from CSI 300 index futures to CSI 300 Spot is always much greater than that from CSI 300 Spot to CSI 300 index, this may not be true in practice since stock market is of high randomness. The NC results showed that the causal influence from CSI 300 index futures to CSI 300 Spot is greater than that from CSI 300 Spot to CSI 300 index by $80 \%$, this may be true in practice. In this way, we actually demonstrate that $\mathrm{NC}$ is better than GC to reveal true causality of one system. Anyway, both methods demonstrated that CSI 300 index futures has a major causal influence in CSI 300 Spot.

\section{Acknowledgements}

This work was funded by National Natural Science Foundation of China under Grant No.61473110, Natural Science Foundation of Zhejiang Province, China, Grant No. Z13F030002, International Science \& Technology Cooperation Program of China, Grant No. 2014DFG12570. Key Lab of Complex Systems Modeling \& Simulation, Ministry of Education, China.

\section{References}

[1] C. W. J. Granger, "Investigating causal relations by econometric models and cross-spectral methods", Econometrica, vol. 37, no. 4, pp.424-438, Aug. 1969.

[2] N. Wiener, The theory of prediction. In E.F. Beckenbach, editor,Modern Mathematics for Engineers, Chap. 8. McGraw-Hill, New York,1956.

[3] M. Ding, Y. Chen, and S. L. Bressler, "Granger causality: Basic theory and applications to neuroscience", in Handbook of Time Series Analysis,B. Schelter, M. Winterhalder, and J. Timmer Eds. Weinheim, Germany:Wiley-VCH, pp. 437-460, 2006.

[4] S. Hu, G. Dai, G. Worrell, Q. Dai, and H. Liang, "Causality analysis of neural connectivity: Critical examination of existing methods and advances of new methods", IEEE Trans Neural Networks, vol. 22, no.6, pp. 829-844, 2011.

[5] H. Cai and X. Li, "Price discovery in the CSI300 stock index futures market in Chinese.2012.

[6] P. Foresti, "Testing for Granger causality between stock prices and economic growth",MPRA Paper No. 2962, posted 26. April 2007.

[7] K.Mshahiro,"Price volatility of storable commodities under rational ecpectations in spot and futures markets",International Economic Review,vol,24,no,2,pp.435-459,1983. 
[8] M. Hernandez and M. Torero, "Examining the dynamic relationship between spot and future prices of agricultural commodities”, IFPRI Discussion Paper 00988, June 2010.

[9] http://www.wind.com.cn/default.aspx. 